\title{
Hard X-ray irradiation of cosmic silicate analogs: structural evolution and astrophysical implications
}

\author{
L. Gavilan ${ }^{1}$, C. Jäger ${ }^{2}$, A. Simionovici ${ }^{3}$, J. L. Lemaire ${ }^{4}$, T. Sabri ${ }^{2}$, E. Foy ${ }^{5}$, S. Yagoubi ${ }^{6}$, T. Henning ${ }^{7}$, \\ D. Salomon ${ }^{8}$, and G. Martinez-Criado ${ }^{8}$
}

\author{
${ }^{1}$ Institut d'Astrophysique Spatiale (IAS), CNRS, Univ. Paris Sud, Université Paris-Saclay, 91405 Orsay, France \\ e-mail: lisseth.gavilan@ias.u-psud.fr \\ ${ }^{2}$ Laboratory Astrophysics and Cluster Physics Group of the Max Planck Institute for Astronomy at the Friedrich Schiller University \\ \& Institute of Solid State Physics, Helmholtzweg 3, 07743 Jena, Germany \\ ${ }^{3}$ Institut des Sciences de la Terre, Observatoire des Sciences de l'Univers de Grenoble, BP 53, 38041 Grenoble, France \\ ${ }^{4}$ Institut des Sciences Moléculaires d'Orsay (ISMO), CNRS, Univ. Paris Sud, Université Paris-Saclay, 91405 Orsay, France \\ ${ }^{5}$ LAPA-IRAMAT, NIMBE, CEA, CNRS, Université Paris-Saclay, CEA Saclay, 91191 Gif-sur-Yvette, France \\ ${ }^{6}$ LEEL, NIMBE, CEA, CNRS, Université Paris-Saclay, CEA Saclay, 91191 Gif-sur-Yvette, France \\ 7 Max-Planck Institute for Astronomy Königstuhl 17, 69117 Heidelberg, Germany \\ ${ }^{8}$ European Synchrotron Radiation Facility, 71 avenue des Martyrs, 38000 Grenoble, France
}

Received 6 November 2015 / Accepted 19 January 2016

\begin{abstract}
Context. Protoplanetary disks, interstellar clouds, and active galactic nuclei contain X-ray-dominated regions. X-rays interact with the dust and gas present in such environments. While a few laboratory X-ray irradiation experiments have been performed on ices, $\mathrm{X}$-ray irradiation experiments on bare cosmic dust analogs have been scarce up to now.

Aims. Our goal is to study the effects of hard X-rays on cosmic dust analogs via in situ X-ray diffraction. By using a hard X-ray synchrotron nanobeam, we seek to simulate cumulative X-ray exposure on dust grains during their lifetime in these astrophysical environments and provide an upper limit on the effect of hard X-rays on dust grain structure.

Methods. We prepared enstatite $\left(\mathrm{MgSiO}_{3}\right)$ nanograins, which are analogs to cosmic silicates, via the melting-quenching technique. These amorphous grains were then annealed to obtain polycrystalline grains. These were characterized via scanning electron microscopy (SEM) and high-resolution transmission electron microscopy (HRTEM) before irradiation. Powder samples were prepared in X-ray transparent substrates and were irradiated with hard X-rays nanobeams $(29.4 \mathrm{keV})$ provided by beamline ID16B of the European Synchrotron Radiation Facility (Grenoble). X-ray diffraction images were recorded in transmission mode, and the ensuing diffractograms were analyzed as a function of the total X-ray exposure time.

Results. We detected the amorphization of polycrystalline silicates embedded in an organic matrix after an accumulated X-ray exposure of $6.4 \times 10^{27} \mathrm{eV} \mathrm{cm}^{-2}$. Pure crystalline silicate grains (without resin) do not exhibit amorphization. None of the amorphous silicate samples (pure and embedded in resin) underwent crystallization. We analyze the evolution of the polycrystalline sample embedded in an organic matrix as a function of X-ray exposure.

Conclusions. Loss of diffraction peak intensity, peak broadening, and the disappearance of discrete spots and arcs reveal the amorphization of the resin embedded (originally polycrystalline) silicate sample. We explore the astrophysical implications of this laboratory result as an upper limit to the effect of X-rays on the structure of cosmic silicates.
\end{abstract}

Key words. astrochemistry - dust, extinction - evolution - methods: laboratory: solid state - methods: analytical

\section{Introduction}

X-rays are an important component of the radiation field of young stellar objects (YSOs). In the pre-main sequence phase, emission is dominated by intense flares, called catastrophic flares (Feigelson et al. 2003; Feigelson 2005), which can reach an X-ray luminosity $\left(L_{\mathrm{X}}\right)$ in the range of $10^{32} \mathrm{erg} \mathrm{s}^{-1}$ within hours. Smaller flares $\left(L_{\mathrm{X}} \sim 10^{31} \mathrm{erg} \mathrm{s}^{-1}\right)$ produced in YSOs and in the modern Sun last a few hours and cycle every few days. When these young stars enter the main sequence, the X-ray luminosity is in the range of $10^{30} \mathrm{erg} \mathrm{s}^{-1}$ (Shu et al. 2001). In young stars, X-rays dominate extreme ultraviolet (EUV) fluxes up to 100 million years (Myr), and the X-ray/EUV ratio remains within a factor of two for stars as old as 1 Gyr. For today's Sun, this ratio is about 0.25 (Ribas et al. 2005). Thus it is expected that such energetic X-ray radiation, dominating UV photons, contributes to the processing of circumstellar materials, mostly illuminated by young T Tauri type stars. Shu et al. (2001) studied the melting capabilities of observed hard $\mathrm{X}$-ray flares in protostars and suggest that vapor-phase condensation of flare-evaporated material can lead to small refractory solids.

Amorphous silicates are abundant in primitive extraterrestrial materials and most astrophysical environments (Henning 2010). The Infrared Space Observatory (ISO) has revealed that $>99 \%$ of silicates are amorphous in the interstellar medium (ISM) with a crystalline fraction of $0.2 \% \pm 0.2 \%$ by mass (Kemper et al. 2004). This is lower than the crystalline fraction observed in the circumstellar envelopes of evolved stars, the main contributors of dust to the ISM. Crystalline silicates are also found in comets and interplanetary dust particles (IDPs) (Molster \& Kemper 2005; Nuth \& Johnson 2006). Glass with embedded metals and sulfide (GEMS) in IDPs contain silicates in amorphous and crystalline form, and it was suggested that 

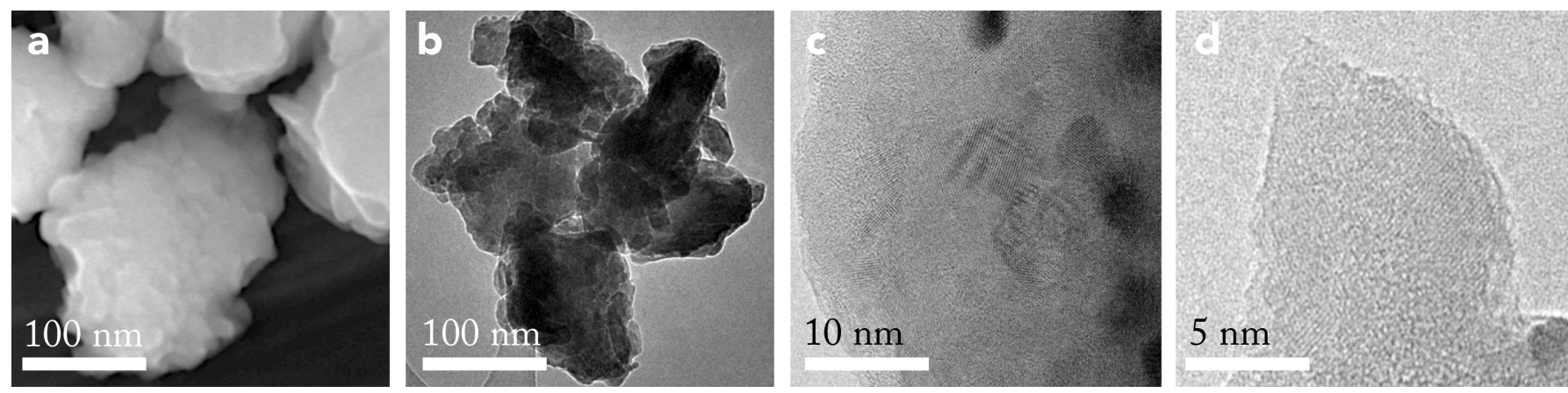

Fig. 1. $\mathrm{MgSiO}_{3}$ crystals before irradiation prepared by quenching or melting. a) FESEM image showing the agglomerated grains surface; b) HRTEM image showing the stacking of agglomerated grains (100 nm scale); c) HRTEM image showing crystalline grains in an amorphous matrix (10 nm scale); d) HRTEM image showing a single crystalline grain (5 nm scale).

these crystalline silicates became amorphous by radiation damage (Bradley 1994). Indeed, depending on their astrophysical environment, dust grains can be altered by several energetic mechanisms, both thermal and non-thermal. Thermal annealing, in which silicates are heated up to $1000 \mathrm{~K}$, can crystallize dust grains. Non-thermal sources include photons, electrons, and ions. Molster et al. (1999) report observations of highly crystalline silicate dust in the disks surrounding binary red-giant stars, which are produced in amorphous form in their outer atmospheres. Since the temperatures in these disks are too low for grains to undergo thermal annealing, they propose that a nonthermal mechanism is responsible for the crystallization. We propose X-ray irradiation as a possible non-thermal mechanism acting on the structure of cosmic silicates.

Hard X-rays permeate several astrophysical environments where silicate and other types of dust are present, such as protoplanetary disks surrounding young stars, planetary nebulae, active galactic nuclei (AGN), and any X-ray-dominated region (XDR). In XDRs, photons can penetrate deeply within icy dust mantles, activating a bulk chemistry that competes with surface processes, such as UV photons and electron irradiation. We expect X-rays to have an effect on the structure of dust grains owing to thermal transfer and/or charge accumulation during their lifetime in XDRs.

Few laboratory experiments exist that study the interaction of $\mathrm{X}$-rays with cosmic dust analogs. Dust irradiation experiments have mostly dealt with ices (e.g., Andrade et al. 2010; Ciaravella et al. 2012; Chen et al. 2013). Experiments of X-ray irradiation on dust mineral analogs at room temperature are scarce. Simionovici et al. (2011a,b) studied the effect of X-rays on returned dust grains from the Stardust mission embedded in aerogel, an extremely low density solid $\left(\rho<0.5 \mathrm{~g} \mathrm{~cm}^{-3}\right)$ used for gently stopping hypervelocity $\left(6-10 \mathrm{~km} \mathrm{~s}^{-1}\right)$ grains in space. To estimate the total exposure to X-rays, they considered an upper limit dose that they called the astrophysical limit (AL). This is the estimated X-ray exposure on an interstellar dust grain during its lifetime in a diffuse cloud $\left(\sim 3 \times 10^{7} \mathrm{yr}\right.$, McKee (1989)). In these clouds, dust grains are exposed to a quasi-isotropic diffuse cosmic X-ray background in the $3-300 \mathrm{keV}$ energy range, most likely produced by AGN components, as measured by the HEAO 1 spacecraft (Gruber et al. 1999). Considering the $\mathrm{X}$-ray flux integrated over time (fluence) and neglecting other irradiation sources, the estimated X-ray astrophysical limit is $5 \times 10^{23} \mathrm{eV} \mathrm{cm}^{-2}$. Stardust mission grains embedded in aerogel were irradiated using a synchrotron X-ray nanobeam $(17 \mathrm{keV}$, $10^{10} \mathrm{ph} \mathrm{s}^{-1}, 150 \times 190 \mathrm{~nm}$ ) and showed damage effects (radial smearing) that were mainly attributed to secondary charge accumulation (Simionovici et al. 2011a). We use the AL as a reference number to compare synchrotron exposure (large flux on a short timescale) to the total exposure on an astronomical time scale (low flux on a large timescale). This exposure time does not consider X-ray damage on dust particles because these processes depend on integrated time, X-ray energy, elemental composition of dust, and thickness. Since all experiments presented in this paper were done using the same monochromatic X-ray flux, we consider the total exposure as the main variable.

By using high-flux synchrotron X-rays, we seek to understand the role of hard X-rays in the structure and/or morphology of dust grains in astrophysical X-ray-dominated regions. We compare synchrotron fluences to the X-ray exposures on dust particles during their lifetime in interstellar clouds, circumstellar, and protoplanetary disks. Because of the nanofocused synchrotron fluxes, we can reach astronomical fluences in less than one second (for the ISM) up to $45 \mathrm{~s}$ (for the circumstellar medium, CSM). This paper is organized as follows. In Sect. 2, we describe sample preparation; in Sect. 3, we present the irradiation experiments; in Sect. 4, we describe the results from the $\mathrm{X}$-ray diffraction analysis; in Sect. 5, we discuss the results; in Sect. 6, we present the astrophysical implications of these experiments. Finally, in Sect. 7, we summarize our conclusions.

\section{Preparation of powder enstatite samples}

Samples were prepared by the Laboratory Astrophysics Group in Jena. Enstatite $\left(\mathrm{MgSiO}_{3}\right)$ grains were prepared using the melting-quenching technique (Dorschner et al. 1995). To produce crystalline $\mathrm{MgSiO}_{3}$, magnesium carbonate and silicon dioxide were thoroughly mixed in stoichiometric quantities and poured into a platinum crucible. The powder was heated in a resistance furnace up to a temperature of $1917 \mathrm{~K}$, and the melt was kept for one hour at the same temperature. To produce a homogeneous $\mathrm{MgSiO}_{3}$ glass, the melt was poured through rotating copper rolls accomplishing quenching rates of $\sim 1000 \mathrm{~K} \mathrm{~s}^{-1}$. The resulting amorphous glass splats were powdered and finally annealed at a temperature of $1400 \mathrm{~K}$ for one hour in air to ensure enstatite formation alone. Size distributions smaller than $500 \mathrm{~nm}$ were finally produced by grinding and sedimentation of the annealed crystalline grains in ethanol. The frequently agglomerated grains are characterized by a very broad size distribution ranging from a few tens up to about $400 \mathrm{~nm}$ (as seen by FESEM and HRTEM images in Figs. 1a, b). The individual particles are composed of randomly oriented, nanometer-sized enstatite crystals still embedded in a small fraction of the amorphous $\mathrm{MgSiO}_{3}$ 


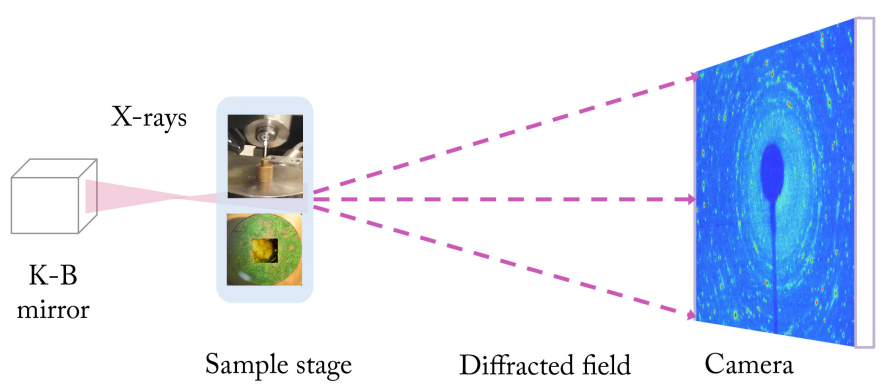

Fig. 2. Schema of the powder diffraction setup at the ID16 beamline at the ESRF. X-rays are focused at the sample, consisting of polycrystalline $\mathrm{MgSiO}_{3}$ powder, placed in a capillary $(\phi=780 \mu \mathrm{m})$ or powder deposited into a $\mathrm{Si}$ box $(\phi=200 \mu \mathrm{m})$ holding a $\mathrm{Si}_{3} \mathrm{~N}_{4}$ membrane. The transmission X-ray diffraction image is recorded behind the sample.

matrix that remained after the final annealing step (see HRTEM micrographs Figs. 1c, d).

$\mathrm{MgSiO}_{3}$ powder samples were deposited on $\mathrm{Si}_{3} \mathrm{~N}_{4}$ membranes that were supported by a Si $0.5 \times 0.5 \mathrm{~mm}$ frame. The $\mathrm{Si}_{3} \mathrm{~N}_{4}$ itself is $50 \mathrm{~nm}$ thick and very fragile when manipulating. It is also quasi-transparent to $\mathrm{X}$-rays and non-diffracting. to insert the powder sample, we used a few drops of $\mathrm{CHCl}_{3}$ for dispersion. In addition, to keep the samples in place on the $\mathrm{Si}_{3} \mathrm{~N}_{4}$ membrane, we used cyanoacrylate $\left(\mathrm{C}_{6} \mathrm{H}_{7} \mathrm{NO}_{2}\right)$, an acrylic resin that rapidly polymerizes in the presence of water (specifically hydroxide ions), forming long, strong chains, thereby joining the bonded surfaces (commercially known as superglue), which is non-diffracting and quasi-transparent to hard X-rays. This had the advantage of keeping the powder samples in place, which are otherwise repelled from the $\mathrm{Si}_{3} \mathrm{~N}_{4}$ film by electrostatic effects. Samples were also deposited in silica capillaries (internal diameter $\phi=780 \mu \mathrm{m}$, and wall thickness $=10 \mu \mathrm{m}$ ).

\section{Irradiation with hard X-rays}

For these experiments we used the recently commissioned ID16B-Nano Analysis beamline at the ESRF synchrotron (Martinez-Criado et al. 2015). The $165 \mathrm{~m}$ long beamline provides nano-focused beams for analytical imaging. During measurements, the spot size at the focal point is $60 \times 80 \mathrm{~nm}^{2}$. The setup is based on Kirkpatrick-Baez (K-B) multilayer, coated Si mirrors, a high-precision piezostage to raster the sample in the beam, and a light microscope to visualize the region of interest on the sample. The setup is schematized in Fig. 2. The penetration depth of the $29.4 \mathrm{keV}$ nanobeam ensures that the full sample thickness is irradiated. Based on a double Si (111) crystal monochromator, we used a $29.4 \mathrm{keV}$ beam with a photon

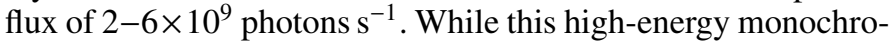
matic beam does not simulate a spectrally broad stellar X-ray spectrum, it provides an upper-limit order-of-magnitude X-ray exposure on dust particles over their lifetime in astrophysical environments.

We used the integration time during the acquisition of the $\mathrm{X}$-ray diffraction image as the exposure time on dust grains, and this allows simultaneous irradiation and recording of the diffraction pattern. If there is continuous irradiation, we could move the diffraction camera (FReLoN CCD) off-sample and, once irradiated, return the camera into the beam path and record the resulting diffraction image. To estimate the optimal sample thickness, we used the X-ray database tool ${ }^{1}$ based on photoabsorption,

http://henke.lbl.gov/optical_constants scattering, and transmission data by Henke et al. (1993). For $\mathrm{MgSiO}_{3}$ grains, we used a density of $3.189 \mathrm{~g} / \mathrm{cm}^{3}$. At $29.4 \mathrm{keV}$, a $200 \mu \mathrm{m}$ thick sample has a transmission of 0.95 , while a $780 \mu \mathrm{m}$ thick sample has a transmission of 0.82 . The absorbed energy per photon is $1.47 \mathrm{keV}$ and $5.29 \mathrm{keV}$, respectively. For powder diffraction, $1 \mu \mathrm{g}$ to $1 \mathrm{mg}$ of sample powder was required. Our initial objective was to irradiate samples until reaching the fluence of $5 \times 10^{23} \mathrm{eV} \mathrm{cm}^{-2}$, expected for a dust grain during its lifetime in an interstellar cloud (the AL limit), as done for Stardust grains embedded in aerogel. With the available synchrotron flux, this was reached after $0.4-1.2 \mathrm{~s}$. We began the irradiation experiments by systematically taking one-second diffraction images to monitor the effect, and subsequently increased to ten-second images in order to better distinguish any further evolution in the diffraction pattern. We continued irradiating samples when small modifications in the diffraction pattern were detected (attenuation of rings, appearance of arcs, disappearance of spots, etc.), which were used as indicators of structural modifications at the nanoscale.

We irradiated the samples beyond the $5 \times 10^{23} \mathrm{eV} \mathrm{cm}^{-2}$ dose (AL limit) in order to verify that an accumulated exposure effect could be detected. In a circumstellar disk, a dust grain exposed to a one-hour catastrophic flare within the reconnection ring receives an exposure of $\sim 10^{25} \mathrm{eV} \mathrm{cm}^{-2}$ and $\sim 10^{22} \mathrm{eV} \mathrm{cm}^{-2}$ for a typical flare (Shu et al. 2001). With the synchrotron nanobeam, these fluences can be achieved after 15 to $45 \mathrm{~s}$, respectively. If we consider the X-ray luminosity for the TW Hydrae (the closest T Tauri star to us) integrated from 0.2 to $2 \mathrm{keV}, L_{\mathrm{X}}=$ $2 \times 10^{30} \mathrm{erg} \mathrm{s}^{-1}$ (Kastner et al. 2002). At ten astronomical units (AU), this amounts to a total exposure of $\sim 10^{26} \mathrm{eV} \mathrm{cm}^{-2}$ in $1 \mathrm{Myr}$ if assuming the X-ray optical depth is $\tau_{\mathrm{X}}=1$ (Gorti \& Hollenbach 2004; Andrade et al. 2010). An equivalent fluence was achieved under $115 \mathrm{~s}$ with the synchrotron nanobeam. While nanoparticles are quasi transparent to hard X-rays, soft $\mathrm{X}$-rays will be more easily absorbed. Thus the exposure dose is only an indicator of the integrated incident energy on the grains, not on the adsorbed energy.

We irradiated four types of powder samples of enstatite $\left(\mathrm{MgSiO}_{3}\right)$ stoichiometry. Sample 1 consisted of crystalline $\mathrm{MgSiO}_{3}$ grains deposited into a resin-embedded $\mathrm{Si}_{3} \mathrm{~N}_{4}$ membrane. Sample 2 consisted of amorphous $\mathrm{MgSiO}_{3}$ grains deposited into a resin embedded $\mathrm{Si}_{3} \mathrm{~N}_{4}$ membrane. Sample 3 consisted of amorphous $\mathrm{MgSiO}_{3}$ grains deposited into a capillary. Sample 4 consisted of crystalline $\mathrm{MgSiO}_{3}$ grains deposited into a capillary.

From these four samples, only Sample 1 showed structural changes during irradiation, as seen in Fig. 3. The interpretation of this result will be explored in the next section. The evolution of the initially polycrystalline sample can be seen in the diffraction patterns. The attenuation of diffraction rings reveals an increasing amorphization. The appearance of some spots shows the preferred orientation due to crystal growth that disappeared under consecutive scans. At an exposure of $3.2 \times 10^{27} \mathrm{eV} \mathrm{cm}^{-2}$ $(2600 \mathrm{~s})$, the diffraction structure (rings, arcs) is largely attenuated. After this exposure time, the sample becomes highly amorphized. A detailed analysis of the structural evolution of this sample will be provided in the next section. Some of the nonevolving diffraction patterns are shown in Fig. 4 as a function of $\mathrm{X}$-ray exposure.

\section{X-ray diffraction analysis}

All samples were kept at a fixed position and diffraction images were taken in transmission mode. Flat-fielded and 

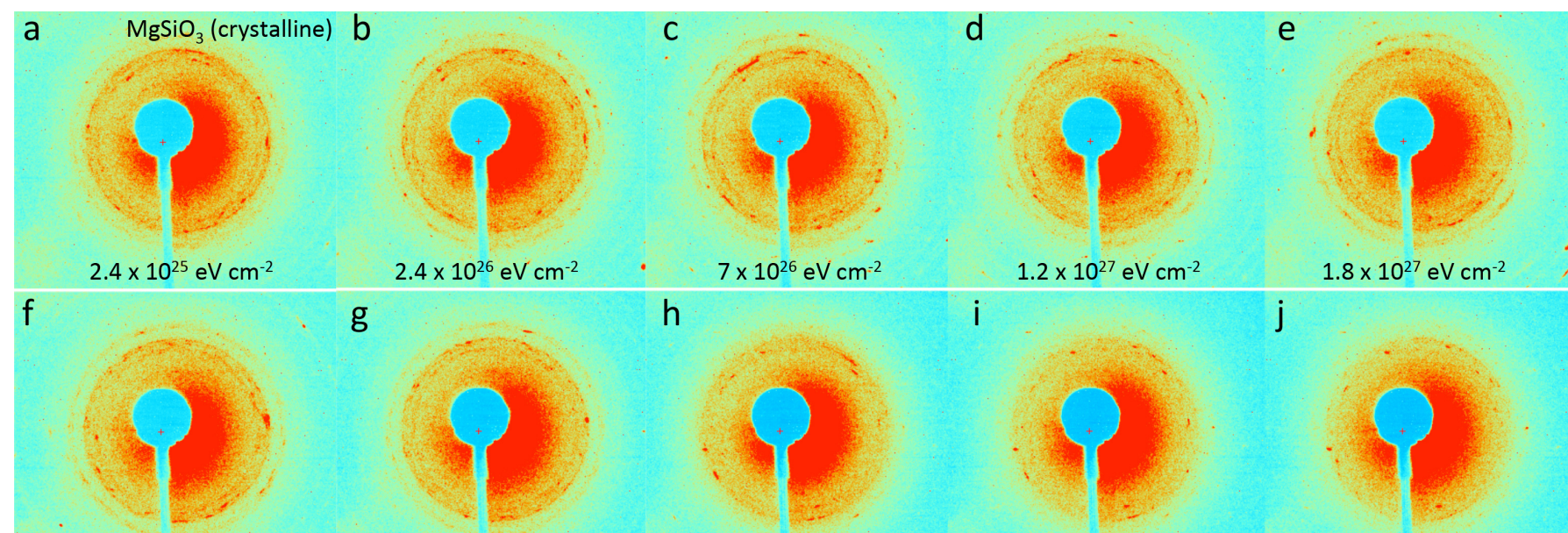

$\mathrm{g}$
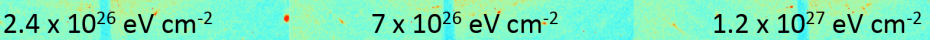

$1.8 \times 10^{27} \mathrm{eV} \mathrm{cm}^{-2}$

h i j

$2.4 \times 10^{27} \mathrm{eV} \mathrm{cm}^{-2}$

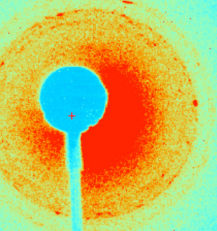

$3 \times 10^{27} \mathrm{eV} \mathrm{cm}^{-2}$

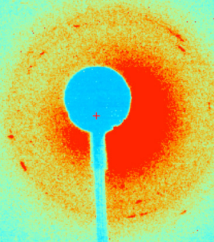

$3.2 \times 10^{27} \mathrm{eV} \mathrm{cm}^{-2}$

$3.6 \times 10^{27} \mathrm{eV} \mathrm{cm}^{-2}$

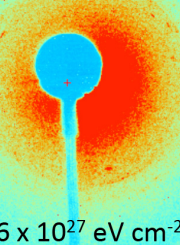

Fig. 3. Diffraction patterns for $\mathrm{MgSiO}_{3}$ crystalline powder samples embedded in the $\mathrm{Si}_{3} \mathrm{~N}_{4}$ substrate at increasing X-ray exposures. a) Crystalline $\left.\left.\mathrm{MgSiO}_{3} ; \mathbf{b}\right)-\mathbf{e}\right)$ diffraction rings and visible arcs; f), g) less rings, few arcs, and spots; $\mathbf{h}$ )-j) no rings, a few spots.
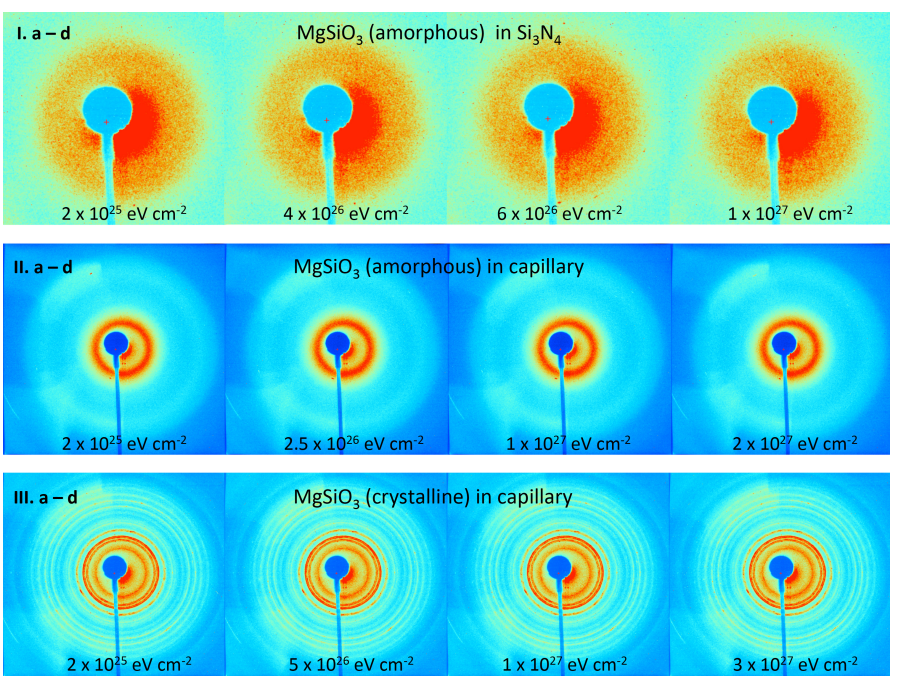

Fig. 4. Samples showing no evolution in their diffraction pattern with increasing X-ray exposure. I) $\mathrm{MgSiO}_{3}$ amorphous grains in a resin embedded $\mathrm{Si}_{3} \mathrm{~N}_{4}$ support. II) $\mathrm{MgSiO}_{3}$ amorphous grains in a capillary. III) $\mathrm{MgSiO}_{3}$ crystalline grains in a capillary.

dark-subtracted diffraction images were processed with Fit2D (Hammersley et al. 1996). The beam-center parameters were refined by using the Bragg diffraction rings. The refined wavelength for irradiation at $29.4 \mathrm{keV}$ was found to be $\lambda=$ $0.42105 \AA$.

In the rest of this section we discuss the diffraction analysis as a function of $\mathrm{X}$-ray exposure for Sample 1, the $\mathrm{MgSiO}_{3}$ grains embedded in the $\mathrm{Si}_{3} \mathrm{~N}_{4}$ membrane, because this is the only sample showing structural changes (Fig. 3). The evolution of the diffractograms with increasing X-ray exposure is shown in Fig. 5. There is a decrease in the intensity of the different peaks as the dose increases in the $2 \theta=9-12^{\circ}$ and $15-18^{\circ}$ ranges, which is seen most clearly for the peak at $2 \theta=8.4^{\circ}$. There is also a clear broadening of all peaks starting from $3 \times 10^{27} \mathrm{eV} \mathrm{cm}^{-2}$. This reveals an amorphization of the polycrystalline grains falling into the X-ray nanobeam spot.

The Jana2006 crystallography software (Petř́ícek et al. 2014) for powder analysis was used for the structural refinement from the $2 \theta$ diffractograms. The peak shapes were adjusted

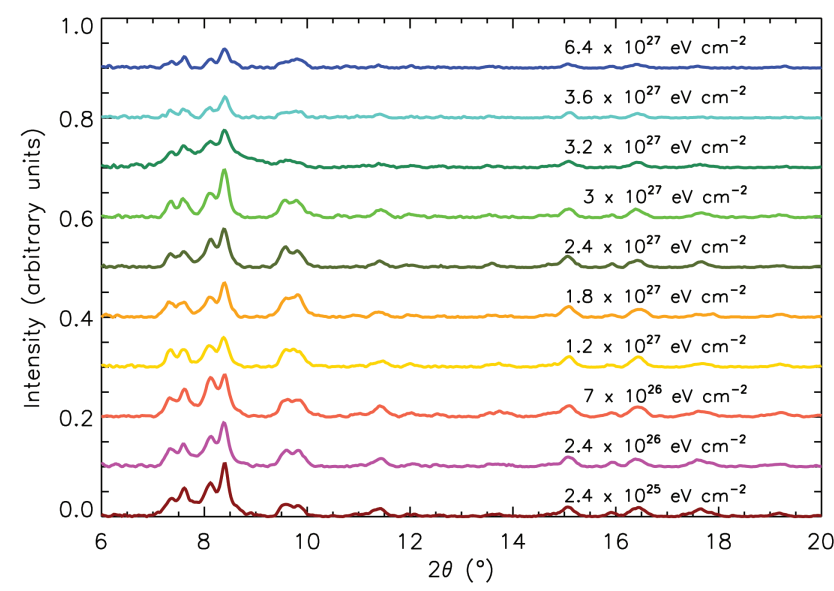

Fig. 5. Evolution of diffraction peaks of $\mathrm{MgSiO}_{3}$ (clinoenstatite) crystalline grains on a resin-embedded $\mathrm{Si}_{3} \mathrm{~N}_{4}$ membrane under X-ray exposures from $2.4 \times 10^{25} \mathrm{eV} \mathrm{cm}^{-2}$ to $6.4 \times 10^{27} \mathrm{eV} \mathrm{cm}^{-2}$.

by Lorentzian functions and a ten-Legendre polynomial was used for background subtraction. At room temperature and ambient pressure, the measured lattice parameters are in good agreement with the literature values for enstatite reported in the Inorganic Crystal Structure Database (ICSD; Ohashi 1984). The powder X-ray diffraction pattern is unambiguously indexed with the monoclinic space group $\mathrm{P} 2{ }_{1} / \mathrm{c}$ and unit cell parameters $a=9.6445(9) \AA, b=8.8264(8) \AA, c=5.1720(4) \AA$, and $\beta=108.790(7)^{\circ}$. The reliability factors after fitting the diffraction peaks (Le Bail refinement, Fig. 6) are $R_{\mathrm{p}}=3.01$ and $\mathrm{w} R_{\mathrm{p}}=5.35$ for the crystalline $\mathrm{MgSiO}_{3}$ sample in the capillary (thickness $=780 \mu \mathrm{m}$, see diffraction patterns II. a-d in Fig. 4). For the crystalline $\mathrm{MgSiO}_{3}$ powder held in a $\mathrm{Si}_{3} \mathrm{~N}_{4}$ window, the optimized reliability factors are $R_{\mathrm{p}} \sim 10$. This is due to the thickness of the capillary sample that is four times larger than the sample in the Si box, increasing the diffraction contrast.

The derived crystallite sizes depend on the instrumental broadening, the monochromator crystals $(2 \times \mathrm{Si} 111)$, the beam divergence, the focusing (K-B mirrors), the shape of the grains, etc. Since we are more interested in the relative evolution of the crystallite structural properties, we assumed that the beam parameters, monochromator, and CCD camera are identical 
L. Gavilan et al.: Hard X-ray irradiation of cosmic silicate analogs: structural evolution and astrophysical implications

Table 1. Fitted crystal parameters for $\mathrm{MgSiO}_{3}$ grains under X-ray irradiation.

\begin{tabular}{cccccccccc}
\hline \hline \multirow{2}{*}{$\begin{array}{c}\text { Exposure } \\
\text { time }[\mathrm{s}]\end{array}$} & \multirow{2}{*}{$\begin{array}{c}\text { Fluence } \\
{\left[\mathrm{eV} \mathrm{cm}{ }^{-2}\right]}\end{array}$} & $a[\AA]$ & $b[\AA]$ & $c[\AA]$ & $\beta\left[\left[^{\circ}\right]\right.$ & $\rho\left[\mathrm{g} \mathrm{cm}^{-3}\right]$ & $\mathrm{D}-\mathrm{S}^{c}$ & ${\mathrm{~W}-\mathrm{H}^{d}}$ & $R_{\mathrm{p}}$ \\
\cline { 3 - 8 } & & 9.6270 & 8.8340 & 5.1800 & 108.3400 & 3.1890 & & & \\
Ref. $^{a}$ & & & \multicolumn{4}{c}{ Lattice parameters } & & & \\
$20^{b}$ & $2.42 \times 10^{25}$ & 9.6445 & 8.8264 & 5.1720 & 108.7902 & 3.1995 & 79.12 & 74.73 & 3.01 \\
20 & $2.42 \times 10^{25}$ & 9.6445 & 8.8264 & 5.1720 & 108.7902 & 3.1995 & 77.97 & 72.38 & 9.67 \\
200 & $2.42 \times 10^{26}$ & 9.6300 & 8.8049 & 5.1542 & 108.6531 & 3.2206 & 86.53 & 89.59 & 11.24 \\
580 & $7.01 \times 10^{26}$ & 9.6276 & 8.7907 & 5.1641 & 108.7908 & 3.2231 & 76.55 & 76.48 & 9.06 \\
2380 & $2.88 \times 10^{27}$ & 9.6097 & 8.8269 & 5.1636 & 108.8866 & 3.2180 & 77.97 & 82.91 & 9.36 \\
2670 & $3.23 \times 10^{27}$ & 9.5725 & 8.8844 & 5.1577 & 109.0414 & 3.2162 & 48.93 & 44.06 & 14.26 \\
\hline
\end{tabular}

Notes. ${ }^{(a)}$ From the Inorganic Crystal Structure Database (ICSD), e.g. Ohashi (1984) for enstatite. ${ }^{(b)}$ Single sample of $\mathrm{MgSiO}_{3}$ grains in the capillary substrate. All other samples in $\mathrm{Si}_{3} \mathrm{~N}_{4}$ windows. ${ }^{(c)}$ Debye-Scherrer method. ${ }^{(d)}$ Williamson-Hall method.

throughout the scans. We estimated the derived error from the fit of all diffraction peaks, which increases naturally thanks to the decrease in diffraction signal. As a lower limit to the crystallite sizes, we used the Debye-Scherrer relation, for which the size is inversely proportional to the full width at half maximum (FWHM) of the diffraction peaks, i.e.,

$D_{v}=\frac{k \lambda}{\beta \cdot \cos (\theta)}$

where $D_{v}$ is the crystallite size, $\beta$ the FWHM in radians, $\theta$ the diffraction angle, and $k=0.94$ the Scherrer constant.

We also used the Williamson-Hall equation integrated in the Jana2006 package for verification. This is given by

$\beta \cdot \cos (\theta)=\frac{\lambda}{D_{v}}+\eta \cdot \sin (\theta)$

where $D_{v}$ is the crystallite size and $\eta$ corresponds to the strain, which is negligible. A few examples of the sizes found through this method are found in Fig. 7. The crystallite sizes found using both methods are in close agreement and are shown in Table 1. We find that grain sizes decrease from $\sim 8 \mathrm{~nm}$ to $4 \mathrm{~nm}$ after an exposure of $6.4 \times 10^{27} \mathrm{eV} \mathrm{cm}^{-2}$ or $40 \mathrm{~min}$ of continuous irradiation. However, considering the larger error of the fitted parameters due to the lower $\mathrm{S} / \mathrm{N}$ of the diffraction peaks (noted in the last column of Table 1) due to loss of crystalline structure, we cannot use the derived crystallite sizes as indicators of the amorphization evolution of the sample. We instead used other diffractogram parameters, such as loss of diffraction peak intensity, peak broadening, and disappearance of discrete spots and arcs, as evidence for the gradual amorphization of this sample. Compared to the beam size of $60 \mathrm{~nm}$, the nanograins no longer behave like crystals (the surface effects become dominant), setting some limits in the classical diffraction laws. A note of caution should be given with the interpretation of the crystallite sizes using the Scherrer equation, a hot topic in the powder diffraction community, e.g. Scardi (2008). The Scherrer and the Williamson-Hall equations constrain the average crystallite size during irradiation, hardly evolving within the error bars.

The lattice parameters from the diffractograms are refined versus irradiation exposures and summarized in Table 1 . With increasing exposure, the increasing amorphization also meant that the $\mathrm{S} / \mathrm{N}$ of the diffraction peaks decreased so that the peak positions and crystallite sizes are dominated by systematic uncertainties. Some of the diffractograms could not be adjusted because the $\mathrm{S} / \mathrm{N}$ was too low and the refinement of the lattice

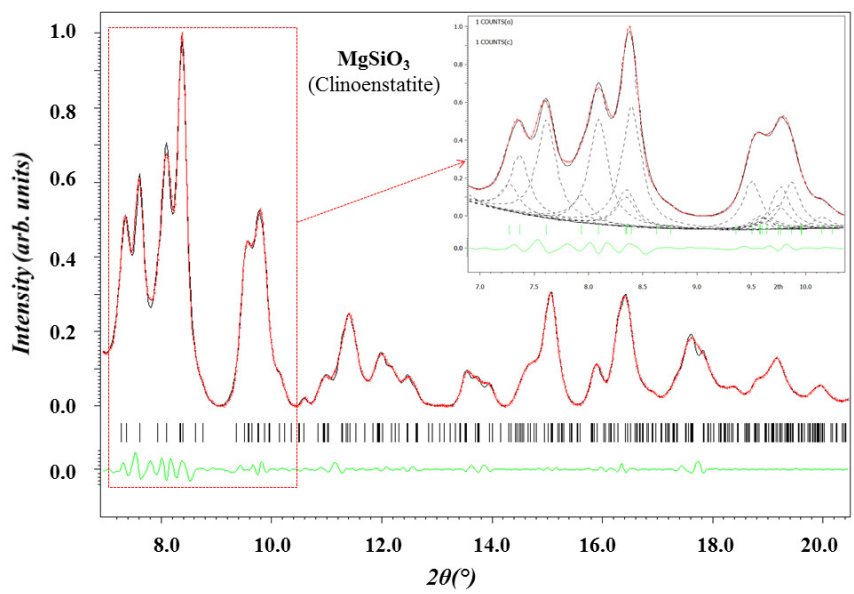

Fig. 6. Calculated scattering intensity profile (black) compared to experimental data (red) collected with synchrotron X-ray diffraction of the crystalline $\mathrm{MgSiO}_{3}$ sample (clinoenstatite) embedded in a capillary.

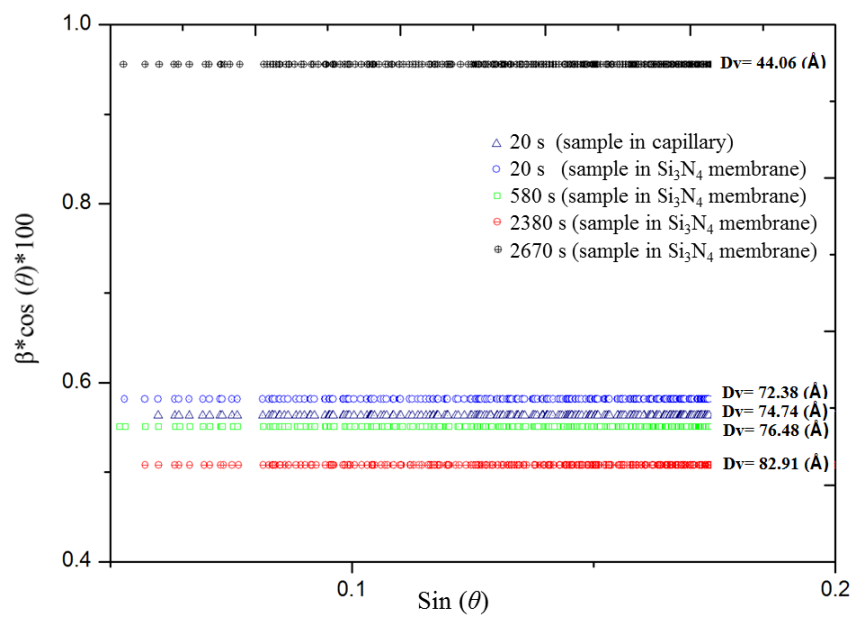

Fig. 7. Crystallite size before and during irradiation of the polycrystalline $\mathrm{MgSiO}_{3}$ sample (clinoenstatite) embedded in a capillary (dark blue triangles) and in the $\mathrm{Si}_{3} \mathrm{~N}_{4}$ membrane.

parameters was degenerate. Analysis of the same powder sample in the $780 \mu \mathrm{m}$ capillary showed the same diffraction peaks and lattice parameters prior to the irradiation series. Diffraction patterns have a higher contrast in the capillary because of the greater thickness $(\phi=780 \mu \mathrm{m})$ compared to the $\mathrm{Si}_{3} \mathrm{~N}_{4}$ substrate 
$(\phi=200 \mu \mathrm{m})$. The average crystallite size obtained from the Williamson-Hall equation for the sample in the capillary is $75 \AA$, which is in good agreement with the size found in the $\mathrm{Si}_{3} \mathrm{~N}_{4}$ substrate.

\section{Discussion}

$\mathrm{X}$-rays on matter promote ionization, excitation, and a population of secondary photoelectrons. These electrons can cause further ionization and elastic interactions with the target atoms, producing point defects and lattice disorder. The deposited energy of a $29.4 \mathrm{keV}$ photon in a single $\phi \sim 8 \mathrm{~nm}$ enstatite grain (see Sect. 4 for the crystallite size determination before irradiation) is negligible; however, for a $200 \mu \mathrm{m}$ or $780 \mu \mathrm{m}$ column of compacted nanometer sized grains, the transmission at $29.4 \mathrm{keV}$ is $\sim 0.953$ or 0.829 , respectively. Most energy incident on the sample is transmitted by Compton scattered X-rays, with an attenuation length of $\sim 3 \mathrm{~mm}$. Only 5 to $18 \%$ of the incident energy is absorbed depending on the sample thickness, as fluorescence of $\mathrm{O}, \mathrm{Mg}$, and $\mathrm{Si}$ with attenuation lengths of $\sim 0.73$, 2.06 , and $2.41 \mu \mathrm{m}$, respectively, and by photoelectrons of energies $\sim 27-29 \mathrm{keV}$ with attenuation lengths in enstatite between 4 and $4.4 \mu \mathrm{m}$ (Katz \& Penfold 1952; Ziaja et al. 2006). When considering a column of compacted grains of macroscopic thicknesses $(200$ or $780 \mu \mathrm{m})$, encased in either $\mathrm{Si}_{3} \mathrm{~N}_{4}$ or in silica (capillaries), the absorbed X-ray energy is fully deposited within the sample.

The geometry and thermal properties of the sample and sample holder should be considered to fully understand the thermal transfer in the sample. The silica capillary and $\mathrm{Si}_{3} \mathrm{~N}_{4}$ membrane are themselves producing secondary electrons. The role of the acrylic resin in the $\mathrm{Si}_{3} \mathrm{~N}_{4}$ membrane samples should also be taken into account. The charge insulation by the resin may enhance charge accumulation further during irradiation. However, previous work has shown that classical thermal transfer models may not be enough to understand the heat transfer of nanoparticles because their thermodynamic stability greatly differs from that of the bulk (Lance Kelly et al. 2003). Ponomarenko et al. (2011) studied radiation-induced melting in X-ray diffractive imaging at the nanoscale by irradiating polymethyl methacrylate (PMMA) samples on a $\mathrm{Si}_{3} \mathrm{~N}_{4}$ window. They argue that the sizes of their nanoparticles were comparable with the characteristic lengths of the heat generation and conduction processes, such as electron or phonon mean free paths. Their calculated rates of temperature rise alone could not explain the melting. Nanometer-sized particles may enhance radiation damage as the photoelectron yield is increased (Lewinski et al. 2009). Such electrons have enough energy to escape from one nanoparticle and penetrate another if the collection is closely packed.

Previous irradiation experiments with silicate analogs (in either amorphous or crystalline form) have mostly involved ion and electron irradiation. Studies with silicate analogs have shown that crystallization of forsterite $\left(\mathrm{MgSiO}_{4}\right)$ grains is possible via thermal annealing at temperatures between $723-873 \mathrm{~K}$ (Fabian et al. 2000). Carrez et al. (2002) show that enstatite grains could be crystallized under $300 \mathrm{keV}$ electron irradiation.

Lemelle et al. (2003) studied the effect of $30 \mathrm{keV}$ electron irradiation on olivine crystals. In their work, they note that with increasing exposures, their nanometer-sized spherules become larger and more irregular in shape. The highest calculated temperature variation due to heat transfer was $40^{\circ} \mathrm{C}$, which is much below the melting point of olivine $\left(1720^{\circ} \mathrm{C}\right.$ at 1 bar). A simple dissipation of the incident energy into heat could not account for the observed damage. The destabilization of olivine was attributed to electrostatic discharges, leading to the breakdown of the dielectric lattice. This occurred when the voltage stress induced by accumulated or trapped charges exceeds the bulk breakdown strength. We interpret the X-ray induced amorphization of our polycrystalline nano-sized enstatite grains embedded in resin, as a result of similar charge build-up processes.

The first ion irradiation experiments of astrophysical interest were performed by Day (1977) and Kraetschmer \& Huffman (1979). Day (1977) exposed olivine to $2 \mathrm{MeV}$ protons, resulting in no alteration. Kraetschmer \& Huffman (1979) showed that olivine was amorphized following exposure to $1.5 \mathrm{MeV}$ $\mathrm{Ne}^{+}$ions. Later on, Bradley (1994) showed that irradiation with light $\mathrm{H}^{+}$and $\mathrm{He}^{+}$ions at 4-20 $\mathrm{keV}$ could amorphize the rim around lunar regolith grains and interplanetary dust particles (IDPs). Demyk et al. (2001) showed that the irradiation with 4-10 $\mathrm{keV} \mathrm{He}{ }^{+}$ions destroys the long-range structure in crystalline olivine and later confirmed this for forsterite, enstatite, and diopside grains (Demyk et al. 2004). Jäger et al. (2003) irradiated enstatite grains with $400 \mathrm{keV} \mathrm{Ar}^{+}$and $50 \mathrm{keV} \mathrm{He}^{+}$ions. Experiments with $30-60 \mathrm{keV} \mathrm{H}^{+}$ions on forsterite also confirmed amorphization (Brucato et al. 2004).

More recently, Bringa et al. (2007) have irradiated forsterite grains with $10 \mathrm{MeV} \mathrm{Xe}^{+3}$ ions. At this energy, $86 \%$ of the total stopping power (energy loss per unit path length) is electronic $\left(4.5 \mathrm{keV} \mathrm{nm}^{-1}\right)$, decreasing with penetration depth as the ion slows down. Electronic energy loss $S_{\mathrm{E}}$ (or electronic stopping power $S_{\mathrm{E}}$ ) causes the breaking of bonds, ionization, and annealing effects. Nuclear energy loss $S_{\mathrm{N}}$ causes knock-on processes and therefore displacements of atoms in the target, which consequently produces amorphization. For heavier ions at similar energy, the fraction of nuclear energy deposition increases (Ziegler et al. 2010). Very energetic ions ( $E \geq 10 \mathrm{MeV})$ also have low $S_{\mathrm{N}} / S_{\mathrm{E}}$ ratios, even though they are heavy. With X-rays, the main interactions remains electronic (inducing core electron excitation/ionization), and their effect on matter is more comparable to that of swift heavy ions than lighter ionic species.

\section{Astrophysical implications}

X-rays are generated by many astrophysical sources, including massive stars, accreting neutron stars, shocks, supernova, and AGN, among others. These X-rays will then interact with the surrounding material mainly composed of gas and dust grains. The launch of the Chandra X-ray Observatory and the XMM-Newton Observatory in 1999 opened a new era in X-ray astronomy by revealing an abundance of X-ray-dominated regions in the cold and hot universe (Paerels \& Kahn 2003).

The properties of dust grains will depend on their astrophysical environment. Dust grains in the interstellar medium are on average isolated $0.1 \mu \mathrm{m}$ sized and quasi-transparent to hard $\mathrm{X}$-rays. Dust grains in protoplanetary disks can grow to $\mathrm{cm}$ sizes via coagulation by the time the central star becomes optically visible (Natta et al. 2007). In these regions, larger dust grains are able to absorb hard X-rays and undergo alteration. Protoplanetary disks are known to have strong X-ray fields arising from the central young stars (Feigelson et al. 2003). Gorti \& Hollenbach (2009) show that X-rays play a role on the disk mass-loss rates via the indirect effect of raising the degree of ionization in the disk and increasing the efficiency of FUV-induced grain photoelectric heating, and in cases with high X-ray luminosities, promoting the formation of gaps in the inner $r \sim 10 \mathrm{AU}$ disk.

Watson et al. (2009) studied a large sample of protoplanetary disks in the Taurus-Auriga young cluster and found that, 
while crystalline silicates are confined to small radii, $r \lesssim 10 \mathrm{AU}$, there is no correlation of the crystalline mass fraction with stellar mass, luminosity, or accretion rate, or even with disk factors (mass, disks/star mass ratio). They suggest that X-ray heating may be dominating the heating and annealing of the grains or that another process must be at work within the disks to erase the correlations they produce (such as giant planet formation and migration). Glauser et al. (2009) examined the first hypothesis by studying the link between the X-ray luminosity and the Xray hardness of the central object of $42 \mathrm{~T}$ Tauri stars (class II) with the crystalline mass fraction of the circumstellar dust. They found a significant anti-correlation for 20 objects within an age range of $\sim 1$ to $4.5 \mathrm{Myr}$. They determined that fluxes around $1 \mathrm{AU}$ and ion energies of the present solar wind are sufficient to amorphize the upper layer of dust grains very efficiently, leading to an observable reduction of the crystalline mass fraction of the circumstellar sub-micron-sized dust. This effect could also erase other relations between crystallinity and disk/star parameters such as age or spectral type. More recently, Cleeves et al. (2014) have studied the primary ionizing agents in the TW Hya protoplanetary disks: cosmic rays and X-rays. They found that the emission spectra due to $\mathrm{HCO}^{+}$and $\mathrm{N}_{2} \mathrm{H}^{+}$is best fitted by moderately hard $\mathrm{X}$-rays, dominating incident cosmic rays.

In light of these findings, two competing mechanisms might be at the origin of the alteration of circumstellar dust. Depending on the age of the central star, X-rays may amorphize grains efficiently, while thermal annealing near the star will crystallize dust grains. Although grain growth could play a role in the disappearance of crystalline features in the mid-infrared, the coagulation of crystalline grains into aggregates is possible. Crystalline silicates observed in disks are significantly smaller than amorphous grains (Juhász et al. 2010) and could assemble into larger amorphous aggregates (Watson et al. 2009; Merín et al. 2007). In this way, crystalline silicate bands will appear as if the crystals were isolated (Min et al. 2008). X-rays can transform the crystals in the aggregates into amorphous grains so that crystalline spectral features are lost. Further observations from the mid-infrared to the millimeter, models including grain size distributions, and laboratory experiments are all needed to distinguish between grain growth or coagulation and amorphization or annealing in protoplanetary disks.

From all our X-ray irradiated samples, only the crystalline enstatite grains embedded in cyanoacrylate, an organic resin, underwent amorphization. The free crystalline grains in the capillary (without resin) did not undergo amorphization. In addition, none of the amorphous silicate samples evolved: neither in the resin-embedded Si box nor in the capillary holder. We interpret this by the fact that free sub-micron-sized grains are quasi-transparent to hard X-rays. Dust grains in the interstellar medium, where grains are sub-micron-sized and isolated, will probably not absorb hard X-rays as long as they are free. On the other hand, the sample that did undergo amorphization was embedded in an organic matrix, acting as a coagulation source, increasing the volume of the sample and allowing charge build-up by secondary photoelectrons. More experiments with different organic materials will be required to explore the role of organics in coagulation and charge build-up under irradiation. Organic and mineral admixtures are present in space, as shown by a study of returned IDPs by Bradley et al. (2005). They show that GEMS contain amorphous silicates surrounded by carbon matter (a mixture of inorganic and organic carbon), with isotopic ratios signaling its interstellar origin. Indeed, during the passage from cold dark clouds to protoplanetary disks, molecules freeze out from the gas phase onto dust grain surfaces, producing icy mantles (Bergin \& Tafalla 2007). Under irradiation (from photons, ions, or electrons), these icy mantles can produce organic residues on mineral grain surfaces. These organic residues can form in molecular clouds, but also in protoplanetary disks and protostellar cores. Protoplanetary disks contain silicates but also carbon dust (Lisse et al. 2006; Tielens 2008) and ices (Carr \& Najita 2008). Organic solids can play a role in the amorphization of silicate-organic aggregates in the X-ray-dominated regions of protoplanetary disks via charging of the particles upon X-ray irradiation.

\section{Conclusions}

Hard X-rays are present in several astrophysical environments. The goal of this study is to simulate the exposure of cosmic dust to hard X-rays and understand the potential effects on the dust grain structure. To do this, we prepared cosmic dust grain analogs: crystalline and amorphous $\mathrm{MgSiO}_{3}$ grains with an average size of $\sim 8 \mathrm{~nm}$. We irradiated these grains with hard X-ray nanobeams produced at the European Synchrotron Radiation Facility (Grenoble) at beamline ID16B-NA and followed their evolution in situ via $\mathrm{X}$-ray diffraction. While irradiation with the monochromatic beam at $29.4 \mathrm{keV}$ gives an upper limit to the cumulative effect of hard X-rays, it does not simulate realistic stellar fluxes in these astrophysical environments. Future experiments are planned with broad spectrum and soft X-ray sources that will complement this work.

An evolution of the structure of crystalline grains embedded in an organic resin toward amorphization was detected after analysis of their diffractograms. The net amorphization of the grains was attributed to charging effects enhanced by the organic matrix, increasing the effective volume of the sample. The reverse process, crystallization of amorphous silicates, was not detected for equivalent X-ray exposures. None of the free samples (without cyanoacrylate) showed any alteration, a result applicable to the interaction of hard X-rays with isolated submicron dust grains in the interstellar medium. Our results manifest the capacity of hard X-rays to modify the structure of agglomerated nanoparticles in higher density regions irradiated by young stars such as protoplanetary disks, where grains can coagulate into $\mathrm{cm}$ sizes and beyond. The results of these high-exposure $\mathrm{X}$-ray irradiation experiments have important consequences for analysis of microscopic mineral samples with synchrotron radiation. In particular, the use of highly insulating materials such as aerogel could affect the analysis of curated dust return samples. High fluences would then have to be carefully limited.

Acknowledgements. We acknowledge the European Synchrotron Radiation Facility (ESRF Grenoble) for providing synchrotron radiation beam time (proposal No. HC-1709), and we thank the beamline scientists of beam line ID16B. We thank Gabriele Born (Astrophysikalisches Institut Jena) for her assistance in sample preparation.

\section{References}

Andrade, D. P. P., Rocco, M. L. M., \& Boechat-Roberty, H. M. 2010, MNRAS, 409, 1289

Bergin, E. A., \& Tafalla, M. 2007, ARA\&A, 45, 339

Bradley, J. P. 1994, Science, 265, 925

Bradley, J., Dai, Z. R., Erni, R., et al. 2005, Science, 307, 244

Bringa, E. M., Kucheyev, S. O., Loeffler, M. J., et al. 2007, ApJ, 662, 372

Brucato, J. R., Strazzulla, G., Baratta, G., \& Colangeli, L. 2004, A\&A, 413, 395

Carr, J. S., \& Najita, J. R. 2008, Science, 319, 1504

Carrez, P., Demyk, K., Cordier, P., et al. 2002, Meteoritics 37, 1599

Chen, Y.-J., Ciaravella, A., Muñoz Caro, G. M., et al. 2013, ApJ, 778, 162

Ciaravella, A., Jiménez-Escobar, A., Muñoz Caro, G. M., et al. 2012, ApJ, 746 L1 
Cleeves, L. I., Bergin, E. A., \& Adams, F. C. 2014, ApJ, 794, 123

Day, K. L. 1977, MNRAS, 178, 49

Demyk, K., Carrez, P., Leroux, H., et al. 2001, A\&A, 368, L38

Demyk, K., d'Hendecourt, L., Leroux, H., Jones, A. P., \& Borg, J. 2004, A\&A, 420, 233

Dorschner, J., Begemann, B., Henning, T., Jaeger, C., \& Mutschke, H. 1995, A\&A, 300, 503

Fabian, D., Jäger, C., Henning, T., Dorschner, J., \& Mutschke, H. 2000, A\&A, 364,282

Feigelson, E. D. 2005, in 13th Cambridge Workshop on Cool Stars, Stellar Systems and the Sun, eds. F. Favata, G. A. J. Hussain, \& B. Battrick, ESA SP, 560,175

Feigelson, E. D., Gaffney, III, J. A., Garmire, G., Hillenbrand, L. A., \& Townsley, L. 2003, ApJ, 584, 911

Glauser, A. M., Güdel, M., Watson, D. M., et al. 2009, A\&A, 508, 247

Gorti, U., \& Hollenbach, D. 2004, ApJ, 613, 424

Gorti, U., \& Hollenbach, D. 2009, ApJ, 690, 1539

Gruber, D. E., Matteson, J. L., Peterson, L. E., \& Jung, G. V. 1999, ApJ, 520, 124

Hammersley, A. P., Svensson, S. O., Hanfland, M., Fitch, A. N., \& Hausermann, D. 1996, High Pressure Research, 14, 235

Henke, B. L., Gullikson, E. M., \& Davis, J. C. 1993, At. Data 54, 181

Henning, T. 2010, ARA\&A, 48, 21

Jäger, C., Fabian, D., Schrempel, F., et al. 2003, A\&A, 401, 57

Juhász, A., Bouwman, J., Henning, T., et al. 2010, ApJ, 721, 431

Kastner, J. H., Huenemoerder, D. P., Schulz, N. S., Canizares, C. R., \& Weintraub, D. A. 2002, ApJ, 567, 434

Katz, L., \& Penfold, A. S. 1952, Rev. Mod. Phys., 24, 28

Kemper, F., Vriend, W. J., \& Tielens, A. G. G. M. 2004, ApJ, 609, 826

Kraetschmer, W., \& Huffman, D. R. 1979, Ap\&SS, 61, 195

Lance Kelly, K., Coronado, E., Zhao, L. L., \& Schatz, G. C. 2003, J. Phys. Chem. B, 107, 668
Lemelle, L., Beaunier, L., Borensztajn, S., Fialin, M., \& Guyot, F. 2003, Geochim. Cosmochim. Acta, 67, 1901

Lewinski, R., Graf, C., Langer, B., et al. 2009, EPJ ST, 219, 67

Lisse, C. M., VanCleve, J., Adams, A. C., et al. 2006, Science, 313, 635

Martinez-Criado, G., Villanova, J., Tucoulou, R., et al. 2015, J. Synchr. Rad., in press

McKee, C. 1989, in Interstellar Dust, eds. L. J. Allamandola, \& A. G. G. M. Tielens, IAU Symp., 135, 431

Merín, B., Augereau, J.-C., van Dishoeck, E. F., et al. 2007, ApJ, 661, 361

Min, M., Hovenier, J. W., Waters, L. B. F. M., \& de Koter, A. 2008, A\&A, 489, 135

Molster, F., \& Kemper, C. 2005, Space Sci. Rev., 119, 3

Molster, F. J., Yamamura, I., Waters, L. B. F. M., et al. 1999, Nature, 401, 563

Natta, A., Testi, L., Calvet, N., et al. 2007, Protostars and Planets V, 767

Nuth, J. A., \& Johnson, N. M. 2006, Icarus, 180, 243

Ohashi, Y. 1984, Phys. Chem. Miner., 10, 217

Paerels, F. B. S., \& Kahn, S. M. 2003, ARA\&A, 41, 291

Petříček, V., Dušek, M., \& Palatinus, L. 2014, Zkri, 229, 345

Ponomarenko, O., Nikulin, A., Moser, H., Yang, P., \& Sakata, O. 2011, J. Synchr. Rad., 18, 580

Ribas, I., Guinan, E. F., Güdel, M., \& Audard, M. 2005, ApJ, 622, 680

Scardi, P. 2008, Z. Kristallogr. Supp., 2008, 101

Shu, F. H., Shang, H., Gounelle, M., Glassgold, A. E., \& Lee, T. 2001, ApJ, 548, 1029

Simionovici, A., Allen, C., Bajt, S., et al. 2011a, in Lunar and Planetary Inst. Technical Report, 42, 2812

Simionovici, A. S., Allen, C., Bajt, S., et al. 2011b, Meteoritics, 74, 5517

Tielens, A. G. G. M. 2008, ARA\&A, 46, 289

Watson, D. M., Leisenring, J. M., Furlan, E., et al. 2009, ApJS, 180, 84

Ziaja, B., London, R. A., \& Hajdu, J. 2006, J. Appl. Phys., 99, 033514

Ziegler, J. F., Ziegler, M., \& Biersack, J. 2010, Nucl. Instrum. Meth. Phys. Res., 268,1818 